\title{
Optimal Sizes and Locations of Piezoelectric Actuators for Curved Panel Sound Sources
}

\author{
Xun Li, Colin H. Hansen and Xiaojun Qiu \\ Department of Mechanical Engineering, The University of Adelaide, Australia
}

\begin{abstract}
Nowadays lead zirconate titanate (PZT) actuators are widely used in active noise and vibration control (ANVC). One of the research areas concerning PZT actuators involves the optimisation of the piezoelectric actuator parameters, including size and location etc., to minimise sound radiated by a vibrating structure. Another practical application of PZT actuators in ANVC is their use to drive a panel which can be utilised as a control source. In this case, it is important to be able to maximise the radiated sound pressure level. This paper presents procedures for optimising sizes and locations of PZT actuators on a resonant curved panel/cavity system to maximise the radiated sound power at a number of discrete frequencies.
\end{abstract}

\section{INTRODUCTION}

In recent years a considerable amount of research has been undertaken in the development of the piezoelectric actuator for active structural acoustic control (ASAC). Some researchers have concentrated on the optimisation of the parameters of the piezoelectric actuators; for instance, the number, sizes, locations and voltages applied to the actuators to minimise either structural vibration or radiated sound power ${ }^{1-6}$. In the work of Wang et al. ${ }^{3}$, a mathematical formulation for the optimisation of the locations of piezoelectric actuators in a feedforward control implementation of ASAC was given. They demonstrated that optimally located piezoelectric actuators can provide a large reduction in the amount of sound power radiated, and are observed to perform better than actuators in arbitrarily chosen locations. Based on the object function selected in their work, Wang et al. found that properly locating one piezoelectric actuator generally gives greater reduction of radiated sound power than using two or three actuators. Recently, Varadan et al. ${ }^{4}$ have reported on the optimisation of the placement of circular disk-shape piezoelectric actuators on a clamped rectangular plate, using the finite element method. They found that the optimal locations of piezoelectric actuators to minimise sound radiation are near the corners rather than the centre of the plate. This is because the edges and corners of flat plates contribute most to the radiated sound power at frequencies below the plate critical frequency, the frequency range of the results reported.

Another application of PZT actuators their use to excite a panel control sources to replace loudspeakers in ANVC (Li et al. ${ }^{7}$ ). In the work of Li et al. ${ }^{7}$, a tuned curved panel sound source for use with active systems for controlling transformer sound was developed. If this kind of sound source is used in the place of loudspeakers, the number of control sources and the complexity of the controller can be reduced substantially. To prevent the interaction and cancellation of the sound radiation from the two sides of the panel, a cavity was coupled to one side of the panel. The reasons for using a curved panel rather than flat panel are twofold: first a curved panel is more easily excited by piezoelectric patch actuators because the bending vibration of the curved panel couples better with the extensional motion of the actuators; and second, it is not possible to adjust the resonance frequencies of the efficient modes of a flat panel very easily.

In this paper, a curved panel with a backing cavity driven by rectangular PZT actuators ${ }^{7}$ has been modelled. Simple optimisation procedures for sizes and locations of the PZT actuators on the panel are given. Because the research described in this paper concerns the design of a resonant curved panel as a sound source, only optimal results for some efficient natural modes are presented. The objective of the optimisation is to maximise the structural response (velocity) of the curved panel vibrating in particular resonant modes.

\section{THEORETICAL MODEL}

\subsection{Responses of Coupled Structural/Acoustic System}

A curved panel coupled with a backing cavity is shown in Fig. 1. A rigid wall cavity is assumed and the panel boundary condition is simply supported.

Due to weak coupling between the structural vibration and acoustic modes (this is allowed when the fluid medium is air), modal coupling theory (Pope ${ }^{8}$ and Hansen and Snyder ${ }^{9}$ ) was used to derive the response expression for a structure/acoustic coupled system. In this case the structural response coupled with an enclosure can be given in matrix form as follows (Hansen and Snyder ${ }^{9}$ ):

$$
\left[\begin{array}{c}
V_{1} \\
V_{2} \\
\vdots \\
V_{m}
\end{array}\right]=\left[\begin{array}{cccc}
Z_{I 1,1} & Z_{I 1,2} & \cdots & Z_{I 1, m} \\
Z_{I 2,1} & \ddots & & \\
\vdots & & \ddots & \\
Z_{I m, 1} & & & Z_{I m, m}
\end{array}\right]^{-1}\left[\begin{array}{c}
\gamma_{1} \\
\gamma_{2} \\
\vdots \\
\gamma_{m}
\end{array}\right],
$$

where $V_{i}=V_{r, s}$ is the structural modal velocity amplitude corresponding to the $r, s$ structural mode resulting from the 